\title{
Weakly Kronecker equivalent number fields and global norms
}

\author{
by \\ MANFRED Lochter (Saarbrücken)
}

1. Introduction and statement of the main results. Class field theory gives a description of the abelian extensions of a local or global field by groups of global norms in the local case and by idele class norms in the global case. In particular, one has (for separable extensions) in the local case (Serre [15, Ch. XI, Prop. 3])

$$
N_{K \mid k}\left(K^{*}\right)=N_{K^{\mathrm{ab}} \mid k}\left(K^{\mathrm{ab} *}\right)
$$

and in the global case (Cassels-Fröhlich [1, exc. 8])

$$
N_{K \mid k}\left(C_{K}\right)=N_{K^{\mathrm{ab}} \mid k}\left(C_{K^{\mathrm{ab}}}\right) .
$$

This shows that in both cases even the Galois extensions are not characterized - among all Galois extensions of $k$ - by their groups of norms.

One aim of this paper is to show that "almost all" extensions (see van der Waerden [18]) $K \mid k$ of number fields are - among all extensions of $k$ characterized by their groups of global norms.

Before the statement of the main results we have to introduce some notation. In the sequel we assume that all fields $K, K^{\prime}, k$ in this paper are algebraic number fields. If $A_{K}$ is one of the arithmetical functors $K^{*}$ multiplicative group, $P_{K}$ group of principal fractional ideals, $I_{K}$ group of fractional ideals, we write

$$
\begin{aligned}
N_{k}\left(A_{K}, A_{K^{\prime}}\right) & :=N A_{K} / N A_{K} \cap N^{\prime} A_{K^{\prime}}, \\
\delta_{k}\left(A_{K}, A_{K^{\prime}}\right) & :=\left(N A_{K}: N A_{K} \cap N^{\prime} A_{K^{\prime}}\right)
\end{aligned}
$$

for the norm class groups and their orders. Here, $N=N_{K \mid k}$ and $N^{\prime}=N_{K^{\prime} \mid k}$ denote the norm from $K$ to $k$ and from $K^{\prime}$ to $k$, respectively. For extensions $K \mid k$ and $K^{\prime} \mid k$ we have the following diagram: 


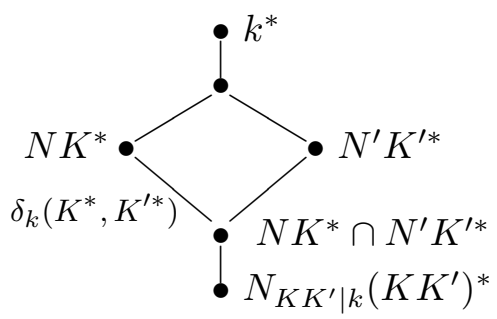

For a finite group $G$ and a subgroup $U$ of $G$ we use the following notation:

$$
U^{G}:=\bigcup_{\sigma \in G} U^{\sigma}, \quad \text { the union of all conjugates of } U \text {. }
$$

$U_{p}$ denotes a Sylow $p$-subgroup of $U$.

Now our main result is:

TheOrem 1. Let $K \mid k$ be an extension of number fields of degree $n$ whose Galois closure $\widetilde{K}$ has Galois group

$$
G(\widetilde{K} \mid k)=S_{n} \quad \text { or } \quad G(\widetilde{K} \mid k)=A_{n>5},
$$

the symmetric group or the alternating group of degree $n(n>5$ in the second case). Then, for any extension $K^{\prime} \mid k$, the following statements are equivalent:

(a) $\delta_{k}\left(K^{*}, K^{\prime *}\right)<\infty$ and $\delta_{k}\left(K^{\prime *}, K^{*}\right)<\infty$.

(b) $K \simeq_{k} K^{\prime}$ (conjugation over $k$ ).

(c) $N_{K \mid k}\left(K^{*}\right)=N_{K^{\prime} \mid k}\left(K^{\prime *}\right)$.

If $K^{\prime} \neq k$, then (a) can be replaced by the equivalent condition

$\left(\mathrm{a}^{\prime}\right) \delta_{k}\left(K^{*}, K^{*}\right)<\infty$.

The proof of Theorem 1 relies on a group theoretic interpretation of the finiteness of the index $\delta_{k}\left(K^{*}, K^{*}\right)$, given in Theorem 2 below, and on the work of R. Guralnick [5]. The theorem follows immediately from [11, Theorem 18] together with Theorem 3.

TheOREM 2. Let $K \mid k$ and $K^{\prime} \mid k$ be extensions of number fields and $N \mid k$ a Galois extension containing $K$ and $K^{\prime}$. In $G=G(N \mid k)$ let $U=G(N \mid K)$ and $U^{\prime}=G\left(N \mid K^{\prime}\right)$ be the respective fixed groups. Then

$$
\delta_{k}\left(K^{*}, K^{\prime *}\right)<\infty \Leftrightarrow \bigcup_{p \mid \# G} U_{p}^{G} \subset \bigcup_{p \mid \# G} U_{p}^{\prime G} .
$$

A proof of this important theorem will be presented in the next section. The discovery of Theorem 2 was inspired by [16]. In this paper L. Stern proved that Galois extensions of number fields are - among all Galois extensions - characterized by their groups of global norms. One year later Stern [17] also proved Theorem 2 (not Theorem 1), but our proof is entirely different. For example L. Stern uses the sophisticated methods of class field 
theory, whereas we only need Chebotarev's density theorem, the finiteness of ideal class numbers and Dirichlet's unit theorem. Although our proof is elementary, a combination with previous results (Lochter [11]) gives effective bounds for the index $\delta_{k}\left(K^{*}, K^{\prime *}\right)$ if it is finite (see Theorem 8):

$$
\delta_{k}\left(K^{*}, K^{\prime *}\right) \text { divides }\left(\mu_{k}: \mu_{k}^{\left(K^{\prime}: k\right)}\right) \cdot\left(K^{\prime}: k\right)^{r_{1}(k)+r_{2}(k)-1} \cdot h_{K^{\prime}} .
$$

A second elementary proof of " $\Rightarrow$ " in Theorem 2, given in Theorem 18, allows a remarkable weakening of the finiteness condition $\delta_{k}\left(K^{*}, K^{\prime *}\right)<\infty$.

In $\S 4$ the same methods are applied in order to gain a full solution of the dual question: When is

$$
\left(\bigcap N_{K_{i} \mid k}\left(K_{i}^{*}\right): N_{F \mid k}\left(F^{*}\right)\right)<\infty,
$$

where $F=K_{1} \ldots K_{n}$ is the composite field? Here we are also able to generalize results of Stern.

Let $K \mid k$ and $K^{\prime} \mid k$ be extensions of number fields. Then $K$ and $K^{\prime}$ are called Kronecker equivalent over $k\left(K \sim_{k} K^{\prime}\right)$ (W. Jehne [6, 7]) iff the Kronecker set $D(K \mid k)$ of those primes of $k$ which have a divisor of first degree in $K$ and $D\left(K^{\prime} \mid k\right)$ coincide up to at most finitely many exceptions (which according to $\mathrm{N}$. Klingen [8] do not exist). Let $P(K)$ denote the set of finite primes of $k$. In the sequel we shall say $\wp \in P(k)$ has decomposition type $\left(f_{1}, \ldots, f_{r}\right)$ in $K \mid k$ iff $\wp$ has exactly $r$ divisors of degrees $f_{1} \leq \ldots \leq f_{r}$ in $K$. In [10] we proved that Kronecker equivalent fields satisfy

$$
\operatorname{gcd}\left(f_{1}, \ldots, f_{r}\right)=\operatorname{gcd}\left(f_{1}^{\prime}, \ldots, f_{s}^{\prime}\right)
$$

for all $\wp \in P(k)$. Here $\wp$ is assumed to have decomposition type $\left(f_{1}, \ldots, f_{r}\right)$ in $K \mid k$ and decomposition type $\left(f_{1}^{\prime}, \ldots, f_{s}^{\prime}\right)$ in $K^{\prime} \mid k$.

In [11] we defined $K$ and $K^{\prime}$ to be weakly Kronecker equivalent over $k$ $\left(K \sim_{s, k} K^{\prime}\right)$ iff (4) holds for (almost) all $\wp \in P(k)$, and proved the following theorem, which contains a characterization of weak Kronecker equivalence:

THEOREM 3. Let $K \mid k$ and $K^{\prime} \mid k$ be extensions of number fields and $N \mid k$ a Galois extension containing $K$ and $K^{\prime}$. In $G=G(N \mid k)$ let $U=G(N \mid K)$ and $U^{\prime}=G\left(N \mid K^{\prime}\right)$ be the respective fixed groups. For a prime number $p$ the following conditions are equivalent:

(a) There is a subset $M \subset P(k)$ of Dirichlet density 0 such that for all $\wp \in P(k) \backslash M$

$$
\min \left(\nu_{p}\left(f_{1}\right), \ldots, \nu_{p}\left(f_{r}\right)\right)=0 \Rightarrow \min \left(\nu_{p}\left(f_{1}^{\prime}\right), \ldots, \nu_{p}\left(f_{s}^{\prime}\right)\right)=0 .
$$

(Here $\nu_{p}$ denotes the $p$-adic valuation.)

(b) For all (or for almost all) $\wp \in P(k)$,

$$
\min \left(\nu_{p}\left(f_{1}^{\prime}\right), \ldots, \nu_{p}\left(f_{s}^{\prime}\right)\right) \leq \min \left(\nu_{p}\left(f_{1}\right), \ldots, \nu_{p}\left(f_{r}\right)\right) .
$$

(c) $U_{p}^{G} \subset U_{p}^{\prime G}$. 
COROLlaRY 4. $K$ and $K^{\prime}$ are weakly Kronecker equivalent over $k$ if and only if $\delta_{k}\left(K^{*}, K^{\prime *}\right)$ and $\delta_{k}\left(K^{*}, K^{*}\right)$ are finite.

In [11] we studied the group theoretic description of weak Kronecker equivalence. The results of this paper can according to Corollary 4 be applied to fields with "almost equal" norm groups. In particular, we showed: Let $\mathcal{N}_{K \mid k}: \mathrm{Cl}(K) \rightarrow \mathrm{Cl}(k)$ be the norm map on ideal class groups. Then $\mathcal{N}_{K \mid k}(\mathrm{Cl}(K))=\mathcal{N}_{K^{\prime} \mid k}\left(\mathrm{Cl}\left(K^{\prime}\right)\right)$.

In our considerations we did not demand $N_{K \mid k}\left(K^{*}\right)=N_{K \mid k}\left(K^{\prime *}\right)$ but allowed the indices $\delta_{k}\left(K^{*}, K^{\prime *}\right)$ and $\delta_{k}\left(K^{\prime *}, K^{*}\right)$ to be finite. For idele class norms, this implies equality:

REMARK 5. Weakly Kronecker equivalent fields satisfy

$$
N_{K \mid k}\left(C_{K}\right)=N_{K^{\prime} \mid k}\left(C_{K^{\prime}}\right) .
$$

Pr o of. (2), together with global class field theory, shows that (5) holds if and only if $K^{\mathrm{ab}}=K^{\prime \mathrm{ab}}$. By [11] weakly Kronecker equivalent fields contain the same Galois extensions of the ground field; in particular, they contain the same abelian extensions of the ground field.

Of course, the converse of Remark 5 is false. The split embedding problem

$$
1 \rightarrow C_{3} \hookrightarrow S_{3} \rightarrow C_{2} \rightarrow 1
$$

has infinitely many solutions with the same maximal-abelian subfield. All these solutions are non-conjugate $S_{3}$-extensions. Hence they are not weakly Kronecker equivalent.

In global class field theory ideles are used. But it is interesting to find a formulation of class field theory which uses $k^{*}$ instead of the idele class group $C_{k}$. For an extension $K \mid k$ let $[K]$ be the weak Kronecker class of $K$. On the set of subgroups of $k^{*}$ one can obviously define an equivalence relation:

$$
H \sim H^{\prime}: \Leftrightarrow\left[\left(H: H \cap H^{\prime}\right)<\infty \wedge\left(H^{\prime}: H \cap H^{\prime}\right)<\infty\right] .
$$

This equivalence relation has the additional property:

$$
\left[H_{1} \sim H_{2} \wedge H_{2} \sim H_{3}\right] \Rightarrow H_{1} \sim H_{2} \cap H_{3} .
$$

Theorem 2 tells us that

$$
\begin{aligned}
i:\{[K] \mid K \text { extension of } k\} & \rightarrow\left\{[H] \mid H \text { subgroup of } k^{*}\right\}, \\
{[K] } & \mapsto\left[N_{K \mid k}\left(K^{*}\right)\right],
\end{aligned}
$$

is injective. But we have not been able to find a description of im $i$. The knowledge of im $i$ would give a description of weak Kronecker classes, in the same way as class field theory describes abelian extensions. One should notice that (by [11]) abelian (even Galois) extensions are contained in all fields of their weak Kronecker class. 
Finally, we mention that weak Kronecker equivalence may also be described in terms of relative Brauer groups (see $\S 5$ ).

2. Proof of Theorem 2 and effective bounds for the norm-index. Let $K \mid k$ be an extension of number fields. Then $I_{K}$ denotes the group of fractional ideals of $K$ and $P_{K}$ the group of principal fractional ideals of $K$. There are norm homomorphisms $N_{K \mid k}: I_{K} \rightarrow I_{k}$ and $N_{K \mid k}: P_{K} \rightarrow P_{k}$. Here, for $\mathcal{P} \in P(K), N_{K \mid k}(\mathcal{P})=\wp^{f}$, where $\wp$ is the prime ideal of $k$ below $\mathcal{P}$ and $f=f_{K \mid k}(\mathcal{P} \mid \wp)$ the residue degree.

As a first important step in the proof of Theorem 2 and of Theorem 8 below we establish:

THEOREM 6. Under the assumptions of Theorem 2 the following assertions are equivalent:

(i) $\bigcup_{p \mid \# G} U_{p}^{G} \subset \bigcup_{p \mid \# G} U_{p}^{\prime G}$.

(ii) $\delta_{k}\left(I_{K}, I_{K^{\prime}}\right)<\infty$.

(iii) $\delta_{k}\left(I_{K}, I_{K^{\prime}}\right)=1$.

(iv) $N_{K \mid k}\left(I_{K}\right) \subset N_{K^{\prime} \mid k}\left(I_{K^{\prime}}\right)$.

Proof. $N_{K \mid k}\left(I_{K}\right)$ is generated by the norms of prime ideals. Hence

$$
N_{K \mid k}\left(I_{K}\right)=\left\langle\wp^{\operatorname{gcd}\left(f_{K \mid k}(\mathcal{P})|\mathcal{P} \in P(K) \wedge \mathcal{P}| \wp\right)} \mid \wp \in P(k)\right\rangle .
$$

From this we deduce

(7) $\quad N_{K \mid k}\left(I_{K}\right) \cap N_{K^{\prime} \mid k}\left(I_{K^{\prime}}\right)=\left\langle\wp^{\operatorname{lcm}\left(\operatorname{gcd}\left(f_{K \mid k}(\mathcal{P})\right), \operatorname{gcd}\left(f_{K^{\prime} \mid k}(\mathcal{P})\right)\right)} \mid \wp \in P(k)\right\rangle$.

Since for every $\wp \in P(k)$,

$\left.\operatorname{gcd}\left(f_{K^{\prime} \mid k}(\mathcal{P})\left|\mathcal{P} \in P\left(K^{\prime}\right) \wedge \mathcal{P}\right| \wp\right)\right) \quad$ divides $\left.\operatorname{gcd}\left(f_{K \mid k}(\mathcal{P})|\mathcal{P} \in P(K) \wedge \mathcal{P}| \wp\right)\right)$

is equivalent to

$$
\operatorname{lcm}\left(\operatorname{gcd}\left(f_{K^{\prime} \mid k}(\mathcal{P})\right), \operatorname{gcd}\left(f_{K \mid k}(\mathcal{P})\right)\right)=\operatorname{gcd}\left(f_{K \mid k}(\mathcal{P})\right),
$$

Theorem 6 follows from Theorem 3 .

A homomorphism $\varphi: A \rightarrow B$ of abelian groups is called a quasiisomorphism if its kernel and cokernel are finite. Then we have

Lemma 7. Let $K \mid k$ and $K^{\prime} \mid k$ be extensions of algebraic number fields. Then:

(a) The natural epimorphism

$$
\psi: N_{k}\left(K^{*}, K^{\prime *}\right) \rightarrow N_{k}\left(P_{K}, P_{K^{\prime}}\right)
$$

is a quasi-isomorphism with kernel order dividing $\left(U_{k}: U_{k} \cap N_{K^{\prime} \mid k}\left(K^{\prime *}\right)\right)$. In particular,

$$
\# \operatorname{ker} \psi \mid\left(\mu_{k}: \mu_{k}^{\left(K^{\prime}: k\right)}\right) \cdot\left(K^{\prime}: k\right)^{r(k)-1},
$$


where $k$ has $r(k)$ infinite primes, unit group $U_{k}$ and group of roots of unity $\mu_{k}$.

(b) The natural homomorphism

$$
\varphi: N_{k}\left(P_{K}, P_{K^{\prime}}\right) \rightarrow N_{k}\left(I_{K}, I_{K^{\prime}}\right)
$$

is a quasi-isomorphism. Its kernel has order dividing the class number $h_{K^{\prime}}$ of $K^{\prime}$.

The proof of Theorem 2 is now obvious. According to Theorem 6 it remains to show that

$$
\delta_{k}\left(K^{*}, K^{\prime *}\right)<\infty \Leftrightarrow \delta_{k}\left(I_{K}, I_{K^{\prime}}\right)<\infty .
$$

But this is clear since the maps $\psi$ and $\varphi$ are both quasi-isomorphisms.

Proof of Lemma 7. (a) It suffices to show

$$
\operatorname{ker} \psi=\frac{N_{K \mid k}\left(K^{*}\right) \cap U_{k} N_{K \mid k}\left(K^{\prime *}\right)}{N_{K \mid k}\left(K^{*}\right) \cap N_{K \mid k}\left(K^{\prime *}\right)} \hookrightarrow U_{k} / U_{k} \cap N_{K \mid k}\left(K^{\prime *}\right) .
$$

For the left hand equality, let $N_{K \mid k}\left(a^{\prime}\right)=a \in N_{K \mid k}\left(K^{*}\right)$ with $\langle a\rangle \in$ $N_{K \mid k}\left(P_{K}\right) \cap N_{K^{\prime} \mid k}\left(P_{K^{\prime}}\right)$. Then

$$
\langle a\rangle=\left\langle N_{K \mid k}\left(a^{\prime}\right)\right\rangle=N_{K \mid k}\left(\left\langle a^{\prime}\right\rangle\right)=N_{K^{\prime} \mid k}\left(\left\langle b^{\prime}\right\rangle\right)=\left\langle N_{K^{\prime} \mid k}\left(b^{\prime}\right)\right\rangle
$$

(cf. W. Narkiewicz [12, p. 151]). This implies $a \in U_{k} N_{K \mid k}\left(K^{\prime *}\right)$, hence $\operatorname{ker} \psi \subset \operatorname{im}($ inc $)$. The converse is plain.

The group on the right of (9) is isomorphic to $U_{k} N_{K \mid k}\left(K^{\prime *}\right) / N_{K \mid k}\left(K^{\prime *}\right)$ into which $\operatorname{ker} \psi$ can be embedded in a natural way. The concrete bound follows from Dirichlet's unit theorem.

(b) The kernel of $\varphi$ can be embedded into $N_{K^{\prime} \mid k}\left(I_{K^{\prime}}\right) / N_{K^{\prime} \mid k}\left(P_{K^{\prime}}\right)$, whose order divides $h_{K^{\prime}}$. As coker $\varphi \simeq N_{K \mid k}\left(I_{K}\right) N_{K^{\prime} \mid k}\left(I_{K^{\prime}}\right) / N_{K \mid k}\left(P_{K}\right) N_{K^{\prime} \mid k}\left(I_{K^{\prime}}\right)$ is finite, the theorem is proved.

Next, we use Theorem 6 and Lemma 7 in order to give effective bounds for the norm index, in case it is finite. These bounds rely on the absence of exceptional primes in Theorem 3 and cannot be derived using Stern's [16], [17] methods.

TheOREM 8. Let $K \mid k$ and $K^{\prime} \mid k$ be extensions of algebraic number fields with finite index $\delta_{k}\left(K^{*}, K^{\prime *}\right)$. Then $\delta_{k}\left(K^{*}, K^{\prime *}\right)$ divides $\left(N_{K \mid k}\left(K^{*}\right) \cap\right.$ $\left.U_{k} N_{K \mid k}\left(K^{\prime *}\right): N_{K \mid k}\left(K^{*}\right) \cap N_{K^{\prime} \mid k}\left(K^{\prime *}\right)\right) \cdot h_{K^{\prime}}$. In particular,

$$
\delta_{k}\left(K^{*}, K^{\prime *}\right) \mid \operatorname{gcd}\left(\# \mu_{k},\left(K^{\prime}: k\right)\right) \cdot\left(K^{\prime}: k\right)^{r(k)-1} \cdot h_{K^{\prime}} .
$$

Here $h_{K^{\prime}}$ can be replaced by the sharper bound \# $\operatorname{ker}\left(\mathcal{N}_{K^{\prime} \mid k}: \operatorname{Cl}\left(K^{\prime}\right) \rightarrow\right.$ $\mathrm{Cl}(k))$. 
Proof. Theorem 6 shows $\delta_{k}\left(I_{K}, I_{K^{\prime}}\right)=1$, hence $\operatorname{ker} \varphi=N_{k}\left(P_{K}, P_{K^{\prime}}\right)$ by Lemma $7(\mathrm{~b})$. Now our assertion follows from Lemma 7, (9) and the fact that $\mu_{k}$ is cyclic. The sharper bound comes from

COROLlary 9. Under the assumptions of Theorem 8, there is a subgroup $R$ of $\operatorname{ker}\left(\mathcal{N}_{K^{\prime} \mid k}: \mathrm{Cl}\left(K^{\prime}\right) \rightarrow \mathrm{Cl}(k)\right)$ and an epimorphism

$$
R \rightarrow N_{k}\left(P_{K}, P_{K^{\prime}}\right) \simeq \frac{N_{K \mid k}\left(K^{*}\right)}{N_{K \mid k}\left(K^{*}\right) \cap U_{k} N_{K \mid k}\left(K^{\prime *}\right)} .
$$

Pr o of. There is an epimorphism

$$
\eta_{1}: \operatorname{ker} \mathcal{N}_{K^{\prime} \mid k} \rightarrow \frac{N_{K^{\prime} \mid k}\left(I_{K^{\prime}}\right) \cap P_{k}}{N_{K^{\prime} \mid k}\left(P_{K^{\prime}}\right)}, \quad \bar{I} \mapsto \overline{N_{K^{\prime} \mid k}(I)},
$$

and a monomorphism

$$
\begin{aligned}
\frac{N_{K \mid k}\left(K^{*}\right)}{N_{K \mid k}\left(K^{*}\right) \cap U_{k} N_{K \mid k}\left(K^{\prime *}\right)} & \simeq N_{k}\left(P_{K}, P_{K^{\prime}}\right) \\
& \simeq \frac{N_{K \mid k}\left(P_{K}\right) N_{K^{\prime} \mid k}\left(P_{K^{\prime}}\right)}{N_{K^{\prime} \mid k}\left(P_{K^{\prime}}\right)} \stackrel{\eta_{2}}{\hookrightarrow} \frac{N_{K^{\prime} \mid k}\left(I_{K^{\prime}}\right) \cap P_{k}}{N_{K^{\prime} \mid k}\left(P_{K^{\prime}}\right)},
\end{aligned}
$$

where $\eta_{2}$ is "inclusion". Here we take notice of $N_{K \mid k}\left(P_{K}\right) N_{K^{\prime} \mid k}\left(P_{K^{\prime}}\right) \subset$ $N_{K^{\prime} \mid k}\left(I_{K^{\prime}}\right) \cap P_{k}$. This inclusion again relies on the absence of exceptional primes in Theorem 3.

In [16] Stern raised the question whether there are non-conjugate fields with $N_{K \mid k}\left(K^{*}\right)=N_{K \mid k}\left(K^{\prime *}\right)$. Later [17] he answered the question affirmatively. Here we present another example.

EXAMPLE 10. Let $p$ be a prime number, $k$ a number field and $K \mid k$ and $K^{\prime} \mid k$ two weakly Kronecker equivalent extensions of degree $p$. Then $N_{K \mid k}\left(K^{*}\right)=N_{K \mid k}\left(K^{\prime *}\right)$.

Pro of. For extensions $K \mid k$ and $K^{\prime} \mid k$ of degree $p$ the following assertions are equivalent (Lochter [11]):

(a) $K$ and $K^{\prime}$ are weakly Kronecker equivalent.

(b) $K$ and $K^{\prime}$ are arithmetically equivalent.

(c) $K$ and $K^{\prime}$ have the same normal closure.

Here $K$ and $K^{\prime}$ are called arithmetically equivalent over $k$ iff for all primes $\wp$ of $k$ the decomposition type of $\wp$ in $K$ and the decomposition type of $\wp$ in $K^{\prime}$ coincide. It is well known that there are infinitely many pairs of non-conjugate arithmetically equivalent fields of prime degree (W. Feit [3]).

The proof of Example 10 is accomplished by the following simple remark, because $p^{2}$ does not divide the order of the symmetric group $S_{p}$. 
REMARK 11. Let $K \mid k$ and $K^{\prime} \mid k$ be extensions of algebraic number fields contained in an extension $F \mid k$. Then the exponent of the group $N_{k}\left(K^{*}, K^{\prime *}\right)$ divides $\operatorname{gcd}\left((F: K),\left(K^{\prime}: K^{\prime} \cap K\right)\right)$.

Proof. Let $i: K \rightarrow F$ be the inclusion map. Then for $a \in K^{*}$,

$$
\begin{aligned}
N_{F \mid k}(i(a)) & =N_{K \mid k}\left(N_{F \mid K}(i(a))\right)=N_{K \mid k}(a)^{(F: K)} \in N_{K \mid k}\left(K^{*}\right) \\
& =N_{K^{\prime} \mid k}\left(N_{F \mid K^{\prime}}(i(a))\right) \in N_{K \mid k}\left(K^{\prime *}\right)
\end{aligned}
$$

and

$$
\begin{aligned}
N_{K \mid k}(a)^{\left(K^{\prime}: K \cap K^{\prime}\right)} & =N_{K \mid k}\left(a^{\left(K^{\prime}: K \cap K^{\prime}\right)}\right)=N_{K \cap K^{\prime} \mid k}\left(N_{K \mid K \cap K^{\prime}}\left(a^{\left(K^{\prime}: K \cap K^{\prime}\right)}\right)\right) \\
& =N_{K \cap K^{\prime} \mid k}\left(N_{K^{\prime} \mid K \cap K^{\prime}}\left(N_{K \mid K \cap K^{\prime}}(a)\right)\right) \\
& =N_{K^{\prime} \mid k}\left(N_{K \mid K \cap K^{\prime}}(a)\right) \in N_{K \mid k}\left(K^{*}\right) \cap N_{K \mid k}\left(K^{\prime *}\right) .
\end{aligned}
$$

It is an interesting question whether weakly Kronecker equivalent fields always satisfy $U_{k} N_{K \mid k}\left(K^{*}\right)=U_{k} N_{K \mid k}\left(K^{*}\right)$ and under which restrictions even $N_{K \mid k}\left(K^{*}\right)=N_{K \mid k}\left(K^{\prime *}\right)$ holds. Here we consider some special cases:

EXAmPle 12. Let $\delta_{k}\left(K^{*}, K^{\prime *}\right)$ be finite and assume $U_{k} \subset N_{K \mid k}\left(K^{\prime *}\right)$ (for example $k=\mathbb{Q},\left(K^{\prime}: \mathbb{Q}\right)$ odd $)$. Then

(a) $\delta_{k}\left(K^{*}, K^{\prime *}\right)$ divides $\#\left(\operatorname{ker}\left(\mathcal{N}_{K^{\prime} \mid k}: \mathrm{Cl}\left(K^{\prime}\right) \rightarrow \mathrm{Cl}(k)\right)\right)$ and the factor group $N_{k}\left(K^{*}, K^{\prime *}\right)$ is an epimorphic image of a subgroup of ker $\mathcal{N}_{K^{\prime} \mid k}$.

(b) If there is an extension $F \mid k$ which contains $K$ and $K^{\prime}$ with $\operatorname{gcd}((F$ : $\left.K),\left(K^{\prime}: K^{\prime} \cap K\right), h_{K^{\prime}}\right)=1$, then $N_{K \mid k}\left(K^{*}\right) \subset N_{K \mid k}\left(K^{\prime *}\right)$.

Proof. Under the condition $U_{k} \subset N_{K \mid k}\left(K^{\prime *}\right)$ there is an isomorphism

$$
N_{k}\left(K^{*}, K^{\prime *}\right) \simeq N_{k}\left(P_{K}, P_{K^{\prime}}\right) .
$$

Thus (a) follows from Corollary 9, and (b) is a consequence of Remark 11.

The same reasoning shows:

EXAMPLE 13. Let $\delta_{k}\left(K^{*}, K^{\prime *}\right)$ be finite. If there is an extension $F$ containing $K$ and $K^{\prime}$ with $\operatorname{gcd}\left((F: K),\left(K^{\prime}: k\right), h_{K^{\prime}}\right)=1$, then $N_{K \mid k}\left(K^{*}\right) \subset$ $U_{k} N_{K \mid k}\left(K^{\prime *}\right)$.

Pr o of. In this case $N_{k}\left(P_{K}, P_{K^{\prime}}\right)$ is trivial.

Next, we consider extensions of $\mathbb{Q}$.

EXAMPLE 14. Let $K \mid \mathbb{Q}$ and $K^{\prime} \mid \mathbb{Q}$ be number fields with finite norm index $\delta_{\mathbb{Q}}\left(K^{*}, K^{\prime *}\right)$.

(a) The norm index divides $2 h_{K^{\prime}}$. If $-1 \in N_{K^{\prime} \mid \mathbb{Q}}\left(K^{\prime *}\right)$ (for example if $\left(K^{\prime}: \mathbb{Q}\right)$ is odd $)$, then $\delta_{\mathbb{Q}}\left(K^{*}, K^{\prime *}\right)$ divides $h_{K^{\prime}}$.

(b) If neither $K$ nor $K^{\prime}$ has a real embedding, then $\delta_{\mathbb{Q}}\left(K^{*}, K^{\prime *}\right)$ divides $h_{K^{\prime}}$. 
Pr o of. We have $\left(N_{K \mid \mathbb{Q}}\left(K^{*}\right) \cap U_{\mathbb{Q}} N_{K^{\prime} \mid \mathbb{Q}}\left(K^{\prime *}\right): N_{K \mid \mathbb{Q}}\left(K^{*}\right) \cap N_{K^{\prime} \mid \mathbb{Q}}\left(K^{\prime *}\right)\right)$ $\leq\left(U_{\mathbb{Q}}: U_{\mathbb{Q}} \cap N_{K^{\prime} \mid \mathbb{Q}}\left(K^{\prime *}\right)\right)=: \Delta$. But $\Delta \in\{1,2\}$ and $\Delta=1$ if $-1 \in$ $N_{K^{\prime} \mid \mathbb{Q}}\left(K^{\prime *}\right)$. Thus (a) is proved. If all norms are positive then obviously $\left(N_{K \mid \mathbb{Q}}\left(K^{*}\right) \cap U_{\mathbb{Q}} N_{K^{\prime} \mid \mathbb{Q}}\left(K^{\prime *}\right): N_{K \mid \mathbb{Q}}\left(K^{*}\right) \cap N_{K^{\prime} \mid \mathbb{Q}}\left(K^{\prime *}\right)\right)=1$. This proves (b).

$A_{5}$-extensions provide an additional example.

EXAMPLE 15. Let $K^{\prime} \mid k$ be an extension of number fields of degree 5 with normal closure $N \mid k$ and $G(N \mid k)=A_{5}$. Then there is a weakly Kronecker equivalent subfield $K$ of degree 10 of $N \mid k$ and the norm groups satisfy:

(a) $N_{K \mid k}\left(K^{*}\right) \subset N_{K \mid k}\left(K^{\prime *}\right)$.

(b) $N_{K \mid k}\left(K^{\prime *}\right) / N_{K \mid k}\left(K^{*}\right) \simeq(\mathbb{Z} / 2 \mathbb{Z})^{n}$ with $n \geq 0$.

Proof. The existence of $K$ is well known (cf. N. Klingen [8]). (a) and (b) are immediate consequences of Remark 11.

$A_{4}$-extensions provide another example of (weakly) Kronecker equivalent fields with known norm factor group (see L. Stern [17]). Here the factor group is related to the Hasse norm principle.

EXAMPLE 16. Let $N \mid k$ be an $A_{4}$-extension of algebraic number fields and denote the cyclic-cubic subfield by $K^{\prime}$. Then $K^{\prime}$ is Kronecker equivalent over $k$ to every subfield $K$ of degree 6 of $N \mid k$ and:

(a) The following two assertions are equivalent:

(i) $N_{K \mid k}\left(K^{*}\right)=N_{K \mid k}\left(K^{*}\right)$.

(ii) The Hasse norm theorem holds for $N \mid K^{\prime}$.

(b) If $\operatorname{gcd}\left(h_{K}, 2\right)=1$ and $k=\mathbb{Q}$, then the following two assertions are equivalent:

(i) $-1 \in N_{K \mid \mathbb{Q}}\left(K^{*}\right)$.

(ii) The Hasse norm theorem holds for $N \mid K^{\prime}$.

Proof. (a) is a direct consequence of Stern [17] and Gurak [4]. Since $\operatorname{gcd}\left(h_{K}, 2\right)=1$, there is an isomorphism

$$
\frac{N_{K \mid k}\left(K^{\prime *}\right)}{N_{K \mid k}\left(K^{*}\right)} \simeq \frac{N_{K \mid k}\left(K^{\prime *}\right) \cap U_{k} N_{K \mid k}\left(K^{*}\right)}{N_{K \mid k}\left(K^{*}\right)} .
$$

If the norm theorem holds for $N \mid K^{\prime}$, then (a) yields $-1=N_{K^{\prime} \mid \mathbb{Q}}(-1) \in$ $N_{K \mid \mathbb{Q}}\left(K^{*}\right)$. Now assume $-1 \in N_{K \mid \mathbb{Q}}\left(K^{*}\right)$. Then $N_{K^{\prime} \mid \mathbb{Q}}\left(K^{\prime *}\right) \subset N_{K \mid \mathbb{Q}}\left(K^{*}\right)$ and consequently $N_{K^{\prime} \mid \mathbb{Q}}\left(K^{\prime *}\right)=N_{K \mid \mathbb{Q}}\left(K^{*}\right)$.

Up to now it is not known whether there are infinite towers of (weakly) Kronecker equivalent fields, but there are towers of arbitrary height (N. Klingen [8]). This fact is the crucial ingredient of: 
EXAmple 17. For every $n \in \mathbb{N}$ there are infinitely many towers $\mathbb{Q} \subset$ $K_{0} \subset K_{1} \subset \ldots \subset K_{n}$, where the fields $K_{i}$ are Kronecker equivalent over $\mathbb{Q}$, $\left(K_{0}: \mathbb{Q}\right)=3^{n}$ and $K_{i+1} \mid K_{i}$ is quadratic. For every such tower exactly one of the following assertions holds.

(a) $\bigwedge_{i \in\{1, \ldots, n\}}\left(N_{K_{0} \mid \mathbb{Q}}\left(K_{0}^{*}\right): N_{K_{i} \mid \mathbb{Q}}\left(K_{i}^{*}\right)\right) \in\{1,2\}$.

(b) $\bigvee_{i \in\{1, \ldots, n\}}\left(N_{K_{0} \mid \mathbb{Q}}\left(K_{0}^{*}\right): N_{K_{i} \mid \mathbb{Q}}\left(K_{i}^{*}\right)\right)=2^{m}, \quad m>1$ and $2^{m-1} d i-$ vides the class numbers of the fields $K_{i}, K_{i+1}, \ldots, K_{n}$.

Proof. The existence of infinitely many towers with the desired properties was proved by N. Klingen [8]. By Remark 11 and Example 14, $N_{K_{i} \mid \mathbb{Q}}\left(K_{i}^{*}\right) / N_{K_{j} \mid \mathbb{Q}}\left(K_{j}^{*}\right)(i \leq j)$ is a 2 -group of order dividing $2 \cdot h_{K_{j}}$.

3. Alternative methods. In this section we present an alternative proof of

$$
\delta_{k}\left(K^{*}, K^{\prime *}\right)<\infty \Rightarrow \bigcup_{p \mid \# G} U_{p}^{G} \subset \bigcup_{p \mid \# G} U_{p}^{\prime G},
$$

which shows that one can weaken the finiteness hypothesis of Theorem 2 .

Theorem 18. Let $K \mid k$ and $K^{\prime} \mid k$ be extensions of algebraic number fields contained in a Galois extension $N \mid k$. Within $G=G(N \mid k)$ let $U$ and $U^{\prime}$ be the respective fixed groups. Let $\left(N_{K \mid k}\left(x_{\iota}\right)\right)_{\iota \in I}$ be a system of representatives for the left cosets of $N_{K \mid k}\left(K^{*}\right) \cap N_{K \mid k}\left(K^{\prime *}\right)$ in $N_{K \mid k}\left(K^{*}\right)$. Let $X$ denote the set of primes

$$
X=\left\{\wp \in P(k)\left|\bigvee_{\iota \in I} \bigvee_{\mathcal{P} \in P(K)} \mathcal{P}\right| \wp \wedge \nu_{\mathcal{P}}\left(\left\langle x_{\iota}\right\rangle_{K}\right) \neq 0\right\}
$$

$\left(\nu_{\mathcal{P}}\right.$ denotes the $\mathcal{P}$-adic valuation). If $X$ has Dirichlet density 0 (for example if $X$ is finite), then $\bigcup_{p \mid \# G} U_{p}^{G} \subset \bigcup_{p \mid \# G} U_{p}^{\prime G}$.

Pr o of. Let $\sigma \in G$ be an element of prime-power order $p^{l}$. We prove:

(10) If $\sigma$ stabilizes a left coset of $U$, then $\sigma$ stabilizes a left coset of $U^{\prime}$.

Then $\bigcup_{p \mid \# G} U_{p}^{G} \subset \bigcup_{p \mid \# G} U_{p}^{\prime G}$, since $U_{p}^{G}$ is the set of elements of $p$-power order which do not act fixed point free on the set $G / U$.

For the proof of (10) we choose $\wp \in P(k) \backslash X$, unramified in $N \mid k$, so that $\sigma=F_{N \mid k}(\mathcal{P})$ is the Frobenius automorphism for a divisor $\mathcal{P}$ of $\wp$ in $N$.

We assume $\wp$ to have exactly $r$ divisors $\mathcal{P}_{1}, \ldots, \mathcal{P}_{r}$ of degree $f_{1} \leq \ldots \leq f_{r}$ in $K$. In $K^{\prime}$ we assume $\wp$ to have exactly $s$ divisors $\mathcal{Q}_{1}, \ldots, \mathcal{Q}_{s}$ of degree $f_{1}^{\prime} \leq \ldots \leq f_{s}^{\prime}$. The residue degrees are the orbit lengths of the action of the cyclic $p$-group $\langle\sigma\rangle$ on $G / U$ respectively on $G / U^{\prime}$, hence they are $p$-powers. By assumption $f_{1}=1$. We have to show $f_{1}^{\prime}=1$. 
$f_{1}=1$ implies $N_{K \mid k}\left(\mathcal{P}_{1}\right)=\wp$. By W. Narkiewicz [12, Theorem 4.3], we can choose $x \in \mathcal{P}_{1}$ so that

$$
z=N_{K \mid k}(x) \in \wp \backslash \wp^{2} .
$$

We already know $z=N_{K \mid k}(x)=N_{K \mid k}\left(x_{\iota}\right) \cdot y$ with $\iota \in I$ and $N_{K^{\prime} \mid k}(\widetilde{y})=$ $y \in N_{K \mid k}\left(K^{*}\right) \cap N_{K \mid k}\left(K^{*}\right)$.

Since $z \in \mathcal{O}_{k} \backslash U_{k}$ the generated ideal is of the form $\langle z\rangle_{k}=\wp \cdot \mathcal{A}, \mathcal{A} \triangleleft \mathcal{O}_{k}$, $\operatorname{gcd}(\wp, \mathcal{A})=1$. Moreover, $\left\langle N_{K \mid k}\left(x_{\iota}\right)\right\rangle_{k}=N_{K \mid k}\left(\left\langle x_{\iota}\right\rangle_{K}\right)$ and $\wp$ are coprime by definition. We calculate

$$
\langle z\rangle_{k}=\left\langle N_{K \mid k}\left(x_{\iota}\right) \cdot N_{K^{\prime} \mid k}(\widetilde{y})\right\rangle=N_{K \mid k}\left(\left\langle x_{\iota}\right\rangle_{K}\right) \cdot N_{K^{\prime} \mid k}\left(\langle\widetilde{y}\rangle_{K^{\prime}}\right),
$$

where $\widetilde{y}$ need not be contained in $\mathcal{O}_{K^{\prime}}$. Nevertheless there is a representation $\langle\widetilde{y}\rangle_{K^{\prime}}=\prod \mathcal{R}_{i}^{\alpha_{i}}, \alpha_{i} \in \mathbb{Z}, \mathcal{R}_{i} \in P\left(K^{\prime}\right)$. Then $N_{K^{\prime} \mid k}\left(\langle\widetilde{y}\rangle_{K^{\prime}}\right)=\prod \mathcal{S}_{i}^{\alpha_{i} \cdot f_{L \mid k}\left(\mathcal{R}_{i} \mid \mathcal{S}_{i}\right)}$, where $\mathcal{S}_{i}$ is the prime of $k$ below $\mathcal{R}_{i}$. Now we consider $\wp$-parts:

$$
\wp=\prod_{\mathcal{R}_{i} \in\left\{\mathcal{Q}_{1}, \ldots, \mathcal{Q}_{s}\right\}} \wp^{\alpha_{i} \cdot f_{K^{\prime} \mid k}\left(\mathcal{R}_{i} \mid \mathcal{S}_{i}\right)} .
$$

This yields

$$
1=\sum_{i=1}^{s} \beta_{i} \cdot f_{i}^{\prime}
$$

with certain $\beta_{i} \in \mathbb{Z} .1 \not \equiv 0(\bmod p)$ finally implies $f_{1}^{\prime}=1$.

Remark. If $K^{\prime} \mid k$ is Galois, then the degrees $f_{i}^{\prime}=f_{1}^{\prime}$ coincide and the same reasoning as above shows $D(K \mid k) \dot{\subset} D\left(K^{\prime} \mid k\right)(\dot{\subset}$ means "contained up to at most finitely many exceptions"). With the help of Bauer's Theorem (see [13]) one shows $K^{\prime} \subset K$. This is an alternative proof of the following result, already contained in [11]:

$$
K^{\prime} \mid k \text { Galois } \wedge \delta_{k}\left(K^{*}, K^{*}\right)<\infty \Rightarrow K^{\prime} \subset K .
$$

The same methods give information on norms of integers:

REMARK 19. Let $K \mid k$ and $K^{\prime} \mid k$ be extensions of number fields.

(a) $U_{k} \cdot N_{K \mid k}\left(\mathcal{O}_{K}\right) \subset U_{k} \cdot N_{K^{\prime} \mid k}\left(\mathcal{O}_{K^{\prime}}\right) \Rightarrow D(K \mid k) \subset D\left(K^{\prime} \mid k\right)$. Note that there are no exceptional primes.

(b) Let $h_{K^{\prime}}=1$. Then we have $U_{k} \cdot N_{K \mid k}\left(\mathcal{O}_{K}\right) \subset U_{k} \cdot N_{K^{\prime} \mid k}\left(\mathcal{O}_{K^{\prime}}\right) \Leftrightarrow$ $D(K \mid k) \subset D\left(K^{\prime} \mid k\right)$.

(c) Let $K \mid \mathbb{Q}$ and $K^{\prime} \mid \mathbb{Q}$ be generated by a root of $f_{1}=X^{7}-7 X+3$ and $f_{2}=X^{7}+14 X^{4}-42 X^{2}-21 X+9$ respectively. Then $K$ and $K^{\prime}$ are not conjugate over $\mathbb{Q}$ and

$$
N_{K \mid \mathbb{Q}}\left(K^{*}\right)=N_{K^{\prime} \mid \mathbb{Q}}\left(K^{\prime *}\right) \quad \text { and } \quad N_{K \mid \mathbb{Q}}\left(\mathcal{O}_{K}\right)=N_{K^{\prime} \mid \mathbb{Q}}\left(\mathcal{O}_{K^{\prime}}\right) .
$$


Proof. (a) Let $\wp \in D(K \mid k)$ and choose $y \in \mathcal{O}_{K}, \omega \in \mathcal{O}_{K^{\prime}}$ and $u \in U_{k}$ with

$$
u \cdot N_{K^{\prime} \mid k}(\omega)=N_{K \mid k}(y) \in \wp \backslash \wp^{2} .
$$

Then $\left\langle N_{K^{\prime} \mid k}(\omega)\right\rangle_{k}=\left\langle N_{K \mid k}(y)\right\rangle_{k}=\wp \cdot \mathcal{A}$ and $\operatorname{gcd}(\wp, \mathcal{A})=1$. Since $\omega$ is an algebraic integer, $\wp$ is contained in $D\left(K^{\prime} \mid k\right)$.

(b) Let $x=N_{K \mid k}(y) \in N_{K \mid k}\left(\mathcal{O}_{K}\right)$ and $y \notin U_{K}$. Then $D(K \mid k) \dot{\subset} D\left(K^{\prime} \mid k\right)$ implies (cf. Lochter [10])

$$
\left\langle N_{K \mid k}(y)\right\rangle_{k}=N_{K \mid k}\left(\langle y\rangle_{K}\right)=N_{K^{\prime} \mid k}(\mathcal{A})=\left\langle N_{K^{\prime} \mid k}(a)\right\rangle_{k}
$$

with a principal ideal $\mathcal{A}=\langle a\rangle$ of $\mathcal{O}_{K^{\prime}}$. Thus $N_{K \mid k}(y) \in U_{k} N_{K^{\prime} \mid K}\left(K^{\prime *}\right)$.

(c) $K$ and $K^{\prime}$ are non-conjugate arithmetically equivalent fields of prime degree [8], hence the first assertion follows from Example 10. Using the computer-algebra-package Kant we have shown $h_{K}=h_{K^{\prime}}=1$, so that the second assertion holds.

4. Finiteness of $N_{K \mid k}\left(K^{*}\right) \cap N_{K^{\prime} \mid k}\left(K^{\prime *}\right): N_{K K^{\prime} \mid k}\left(K K^{\prime *}\right)$. In the last two sections we dealt with the finiteness of $\delta_{k}\left(K^{*}, K^{\prime *}\right)$, but another interesting question is:

Given extensions $K_{1}\left|k, \ldots, K_{n}\right| k$ and $F=K_{1} \ldots K_{n}$, under what conditions is the index $\left(\bigcap_{i=1}^{n} N_{K \mid k}\left(K_{i}^{*}\right): N_{F \mid k}\left(F^{*}\right)\right)$ finite?

In this section we shall give a complete group theoretic and representation theoretic answer to this question. Since the arguments needed for the proof of Theorem 20 below are similar to the ones used for the proof of Theorem 2, we shall not go into details. The interested reader is referred to [9], where the proof of Theorem 20 is carried out.

For the statement of the result we need some notation. For an extension $K \mid k$ of number fields we define

$$
T F(K \mid k)=\left\{\wp \in P(k) \mid \operatorname{gcd}\left(f_{K \mid k}(\mathcal{P})|\mathcal{P} \in P(K) \wedge \mathcal{P}| \wp\right)=1\right\} .
$$

Since two number fields $K\left|k, K^{\prime}\right| k$ are weakly Kronecker equivalent over $k$ if and only if $T F(K \mid k)=T F\left(K^{\prime} \mid k\right)$ (see [11]), the sets $T F(K \mid k)$ are analogues of the sets $D(K \mid k)$ which are used in order to define Kronecker equivalence.

Let $N \mid k$ be a Galois extension containing $K$, and let $G=G(N \mid k)$ and $U=G(N \mid K) . G$ acts on the left cosets of $U$ in $G$; we denote the corresponding permutation character by $\Phi_{U}$. Now the main theorem of this section is:

THeOREM 20. Let $K_{1}\left|k, \ldots, K_{n}\right| k$ be extensions of number fields, and $F=K_{1} \ldots K_{n}$. Within the Galois group $G=G(N \mid k)$ of a Galois extension $N \mid k$ containing $F$, let $U_{i}$ be the corresponding fixed groups. Then the following conditions are equivalent:

(i) $\left(\bigcap_{i=1}^{n} N_{K \mid k}\left(K_{i}^{*}\right): N_{F \mid k}\left(F^{*}\right)\right)<\infty$. 
(ii) $\left(\bigcap_{i=1}^{n} N_{K_{i} \mid k}\left(I_{K_{i}}\right): N_{F \mid k}\left(I_{F}\right)\right)<\infty$.

(iii) $\bigcap_{i=1}^{n} T F\left(K_{i} \mid k\right) \doteq T F(F \mid k)$.

(iv) For almost all $\wp \in P(k)$,

$$
\begin{aligned}
\operatorname{lcm}_{i \in\{1, \ldots, n\}}\left(\operatorname { g c d } \left(f_{K_{i} \mid k}(\mathcal{P}) \mid \mathcal{P} \in\right.\right. & \left.\left.P\left(K_{i}\right) \wedge \mathcal{P} \mid \wp\right)\right) \\
& =\operatorname{gcd}\left(f_{F \mid k}(\mathcal{P})|\mathcal{P} \in P(F) \wedge \mathcal{P}| \wp\right) .
\end{aligned}
$$

(v) $\bigcup_{p \mid \# G}\left(\bigcap_{i=1}^{n} U_{i, p}^{G}\right)=\bigcup_{p \mid \# G}\left(\bigcap_{i=1}^{n} U_{i}\right)_{p}^{G}$.

(vi) For all $p \mid \# G, \bigwedge_{\sigma \in G}\left[\prod_{i=1}^{n} \Phi_{U_{i, p}}(\sigma)>0 \Leftrightarrow \Phi_{\left(\cap_{i=1}^{n} U_{i}\right)_{p}}(\sigma)>0\right]$.

Although we are not going to prove Theorem 20, the following remarks seem to be appropriate.

1. We do not know whether the exceptional primes that are allowed in (iii) and (iv) do actually exist. Thus we are not able to give effective bounds, as good as those given with Theorem 8. Any proof of the nonexistence of exceptional primes would give deep information on the structure of decomposition groups.

2. The analogous condition $D(K \mid k) \cap D\left(K^{\prime} \mid k\right) \doteq D\left(K K^{\prime} \mid k\right)$ is equivalent to $U^{G} \cap U^{\prime G}=\left(U \cap U^{\prime}\right)^{G}$.

3. The assertions of Theorem 20 are not satisfied for all field extensions: Let $N \mid k$ be an $A_{4}$-extension. For two of the conjugate subfields of degree 6 we get $\left(N_{K_{1} \mid k}\left(K_{1}^{*}\right) \cap N_{K_{2} \mid k}\left(K_{2}^{*}\right): N_{N \mid k}\left(N^{*}\right)\right)=\left(N_{K_{1} \mid k}\left(K_{1}^{*}\right): N_{N \mid k}\left(N^{*}\right)\right)=\infty$, since $K_{1}$ and $N$ are not weakly Kronecker equivalent over $k$.

The third remark is also contained in

RemarK 21. Let $K \not \subset K^{\prime}, K^{\prime} \not \subset K$ be given. If $F=K K^{\prime} \mid k$ is Galois and $\left(N_{K \mid k}\left(K^{*}\right) \cap N_{K^{\prime} \mid k}\left(K^{\prime *}\right): N_{F \mid k}\left(F^{*}\right)\right)$ is finite, then the indices $\delta_{k}\left(K^{*}, K^{\prime *}\right)$ and $\delta_{k}\left(K^{\prime *}, K^{*}\right)$ are infinite.

Proof. Assume $\delta_{k}\left(K^{*}, K^{* *}\right)<\infty$. Then $\left(N_{K \mid k}\left(K^{*}\right): N_{F \mid k}\left(F^{*}\right)\right)$ is also finite, i.e. $K$ and $F$ are weakly Kronecker equivalent over $k$. Since $F \mid k$ is Galois, this implies $K=F$ and $K^{\prime} \subset K$.

We now consider the case of two fields. Stern [16, Theorem 1] proved $K \mid k$ Galois $\wedge K^{\prime} \mid k$ Galois $\Rightarrow\left(N_{K \mid k}\left(K^{*}\right) \cap N_{K^{\prime} \mid k}\left(K^{\prime *}\right): N_{K K^{\prime} \mid k}\left(K K^{\prime *}\right)\right)<\infty$. Our group theoretic condition for the finiteness of $\left(N_{K \mid k}\left(K^{*}\right) \cap N_{K^{\prime} \mid k}\left(K^{\prime *}\right)\right.$ : $N_{K K^{\prime} \mid k}\left(K K^{\prime *}\right)$ ) enables us to give a broad generalization of Stern's result (Theorem 22(a)). Theorem 22(b) generalizes Example 4.35 of [9]. Since weakly Kronecker equivalent fields are not linearly disjoint over the ground field, this once more points out a duality between weak Kronecker equivalence and the finiteness of $\left(N_{K \mid k}\left(K^{*}\right) \cap N_{K^{\prime} \mid k}\left(K^{\prime *}\right): N_{K K^{\prime} \mid k}\left(K K^{\prime *}\right)\right)$.

TheOREm 22. Let $K \mid k$ and $K^{\prime} \mid k$ be extensions of number fields. Then

$$
\left(N_{K \mid k}\left(K^{*}\right) \cap N_{K^{\prime} \mid k}\left(K^{\prime *}\right): N_{K K^{\prime} \mid k}\left(K K^{\prime *}\right)\right)<\infty
$$


holds in each of the following cases:

(a) $K$ or $K^{\prime}$ is Galois over $k$.

(b) $K$ and $K^{\prime}$ are linearly disjoint over $k$.

(c) If $\operatorname{gcd}\left((K: k),\left(K^{\prime}: k\right)\right)=1$, then we even have the equality

$$
N_{K \mid k}\left(K^{*}\right) \cap N_{K \mid k}\left(K^{\prime *}\right)=N_{K K^{\prime} \mid k}\left(K K^{\prime *}\right) .
$$

Proof. Let $N \mid k$ be a Galois extension containing $K$ and $K^{\prime}$, and let $G=G(N \mid k), U=G(N \mid K)$, and $V=G\left(N \mid K^{\prime}\right)$. For $p \mid \# G$ we have to show

$$
U_{p}^{G} \cap V_{p}^{G}=(U \cap V)_{p}^{G} .
$$

(a) Assume $K^{\prime} \mid k$ Galois.

" $\subset$ ": Let $x=\sigma u \sigma^{-1}=\tau v \tau^{-1} \in U_{p}^{G} \cap V_{p}^{G}$. Then $u=\sigma^{-1} \tau v \tau^{-1} \sigma \in$ $U \cap V^{G}=U \cap V$. Hence $x \in(U \cap V)_{p}^{G}$. The converse is plain.

(b) Since $K$ and $K^{\prime}$ are linearly disjoint, we have $(V: V \cap U)=(G: U)$, hence $(G: V \cap U)=(G: U)(G: V)$. This is only possible if $G=V U=$ $\{v u \mid v \in V, u \in U\}$ and implies $V^{G}=\bigcup_{\sigma \in G} V^{\sigma}=V^{U}$. Again we have to show (12).

" $\supset$ " is obvious. Now take $x=u v u^{-1} \in U_{p} \cap V_{p}^{G}=U_{p} \cap V_{p}^{U}$. Then $v=u^{-1} x u \in U \cap V$. Hence $x \in(U \cap V)_{p}^{G}$.

(c) The exponent of $N_{K \mid k}\left(K^{*}\right) \cap N_{K \mid k}\left(K^{*}\right) / N_{F \mid k}\left(F^{*}\right)$ divides $\operatorname{gcd}((F$ : $\left.K),\left(F: K^{\prime}\right)\right)$.

5. Relative Brauer groups. Here we give another characterization of weak Kronecker equivalence in terms of relative Brauer groups. Let $K \mid k$ be an extension of number fields. Then the relative Brauer group $B(K \mid k)$ is the kernel of the restriction

$$
r_{K \mid k}: B(k) \rightarrow B(K), \quad[A] \mapsto\left[A \otimes_{k} K\right],
$$

where $B(k)$ denotes the Brauer group of $k$. If $[A] \in B(K \mid k)$, then $K$ is called a splitting field for $[A]$. Let $S_{\infty}(k)$ be the set of infinite primes of $k$. For a prime $\wp \in P(k) \cup S_{\infty}(k)$ there are homomorphisms into the Brauer groups of the completions

$$
B(k) \rightarrow B\left(k_{\wp}\right), \quad[A] \mapsto\left[A \otimes_{k} k_{\wp}\right],
$$

and well known isomorphisms

$$
\operatorname{inv}_{\wp}: B\left(k_{\wp}\right) \simeq \begin{cases}\mathbb{Q} / \mathbb{Z}, & \wp \in P(k), \\ \frac{1}{2} \cdot \mathbb{Z} / \mathbb{Z}, & \wp \text { real }, \\ 0, & \wp \text { complex. }\end{cases}
$$

Further, there is an exact sequence

$$
1 \rightarrow B(K) \stackrel{\varphi}{\hookrightarrow} I(K) \stackrel{\psi}{\rightarrow} \mathbb{Q} / \mathbb{Z} \rightarrow 1,
$$


where $I(K)=\bigoplus_{\wp \in P(k) \cup S_{\infty}(k)} I_{\wp}(k)$ with $I_{\wp}(k)=\mathbb{Q} / \mathbb{Z}$ if $\wp \in P(k), I_{\wp}(k)=$ $\frac{1}{2} \cdot \mathbb{Z} / \mathbb{Z}$ if $\wp$ is real and $I_{\wp}(k)=0$ otherwise. Here $\varphi([A])=\left(\operatorname{inv}_{\wp}\left(\left[A \otimes_{k} k_{\wp}\right]\right)\right)_{\wp}$ and $\psi\left(\left(t_{\wp}\right)_{\wp}\right)=\sum t_{\wp}$.

$K$ is a splitting field for $[A] \in B(k)$ if and only if

$$
\bigwedge_{\wp \in P(k) \cup S_{\infty}(k)} \bigwedge_{\mathcal{P} \mid \wp}\left(K_{\mathcal{P}}: k_{\wp}\right) \cdot \operatorname{inv}_{\wp}\left(\left[A \otimes_{k} k_{\wp}\right]\right)=0 .
$$

For proofs of these classical theorems, the reader is referred to Pierce [14, p. 357, p. 354].

We get the following theorem:

TheOREM 23. Let $K \mid k$ and $K^{\prime} \mid k$ be extensions of number fields contained in a Galois extension $N \mid k$. Let $G=G(N \mid k), U=G(N \mid K)$ and $U^{\prime}=G\left(N \mid K^{\prime}\right)$ be the respective Galois groups. Then for $p \mid \# G$ the following statements are equivalent:

(i) $\left(B(K \mid k)_{p}: B(K \mid k)_{p} \cap B\left(K^{\prime} \mid k\right)_{p}\right)<\infty$.

(ii) $U_{p}^{\prime G} \subset U_{p}^{G}$.

Here $B(K \mid k)_{p}$ denotes the $p$-torsion part of $B(K \mid k)$. A first version of Theorem 23 was proved by Fein, Kantor, and Schacher [2]: $B(K \mid k)_{p}$ is finite if and only if $U_{p}^{G}=G_{p}^{G}$. Later R. Guralnick [5] used (i) $\Rightarrow$ (ii) implicitly without giving a proof. For the readers' convenience, we include a short proof of Theorem 23:

We abbreviate $\operatorname{inv}_{\wp}\left(\left[A \otimes k_{\wp}\right]\right)$ by $\operatorname{inv}_{\wp}([A])$.

(i) $\Rightarrow$ (ii). Let $\left\{\left[A_{1}\right], \ldots,\left[A_{s}\right]\right\}$ be a system of representatives for the cosets of $B(K \mid k)_{p} \cap B\left(K^{\prime} \mid k\right)_{p}$ in $B(K \mid k)_{p}$. We consider the finite set

$$
M=\left\{\wp \in P(k) \cup S_{\infty}(k) \mid \bigvee_{i \in\{1, \ldots, s\}} \operatorname{inv}_{\wp}\left(\left[A_{i}\right]\right) \neq 0\right\} .
$$

For $\sigma \in U_{p}^{\prime}$ we choose $\wp_{0} \neq \wp_{0}^{\prime} \in P(k) \backslash M$, unramified in $N \mid k$, such that $\sigma=F_{N \mid k}\left(\mathcal{P}_{0}\right)=F_{N \mid k}\left(\mathcal{P}_{0}^{\prime}\right)$ for a divisor $\mathcal{P}_{0}$ of $\wp_{0}$ in $N$ and a divisor $\mathcal{P}_{0}^{\prime}$ of $\wp_{0}^{\prime}$ in $N$. Then we define

$p^{i}:=\min \left\{f_{K \mid k}(\mathcal{P})|\mathcal{P} \in P(K) \wedge \mathcal{P}| \wp_{0}\right\}=\min \left\{f_{K \mid k}(\mathcal{P})|\mathcal{P} \in P(K) \wedge \mathcal{P}| \wp_{0}^{\prime}\right\}$.

By (13) there is $[A] \in B(k)_{p}$ with

$$
\operatorname{inv}_{\wp}([A])= \begin{cases}1 / p^{i}, & \wp=\wp_{0}, \\ -1 / p^{i}, & \wp=\wp_{0}^{\prime}, \\ 0, & \text { otherwise. }\end{cases}
$$

By the characterization (14) of splitting fields, $[A] \in B(K \mid k)_{p}$. Thus

$$
[A]=\left[A_{j}\right] \cdot[B] \quad \text { with } j \in\{1, \ldots, s\} \text { and }[B] \in B\left(K^{\prime} \mid k\right)_{p} .
$$


For $\wp \notin M$ we have

$$
\operatorname{inv}_{\wp}([A])=\operatorname{inv}_{\wp}([B]) .
$$

Now (14) and (15) yield

$$
\overline{0}=\operatorname{inv}_{\wp_{0}}([B])=\frac{1}{p^{i}}+\mathbb{Z},
$$

hence $\sigma \in U_{p}^{G}$.

(ii) $\Rightarrow(\mathrm{i})$. Let $U_{p}^{\prime G} \subset U_{p}^{G}$. We choose a system $\left(\left[B_{\iota}\right]\right)_{\iota \in I}$ of representatives for the cosets of $B(K \mid k)_{p} \cap B\left(K^{\prime} \mid k\right)_{p}$ in $B(K \mid k)_{p}$ and fix an unramified $\wp_{0} \in P(k)$ with the property

$$
\begin{aligned}
\min \left\{\nu_{p}\left(f_{K \mid k}(\mathcal{P})\right) \mid \mathcal{P} \in P(K)\right. & \left.\wedge \mathcal{P} \mid \wp_{0}\right\} \\
& =\max \left\{\min \left\{\nu_{p}\left(f_{K \mid k}(\mathcal{P})\right)|\mathcal{P} \in P(K) \wedge \mathcal{P}| \wp\right\}\right\},
\end{aligned}
$$

where $\wp$ runs through the set of those primes of $k$ which are unramified in $N \mid k$.

For fixed $\iota_{0} \in I$ we consider the finite set

$$
M=\left\{\wp \in P(k) \mid \operatorname{inv}_{\wp}\left(\left[B_{\iota_{0}}\right]\right) \neq 0\right\} .
$$

Let $\wp_{0} \neq \wp_{1} \in M$ be unramified in $N \mid k$ with $\operatorname{inv}_{\wp_{1}}\left(\left[B_{\iota_{0}}\right]\right)=\lambda / p^{i}, \lambda \in \mathbb{Z}$, $\operatorname{gcd}(\lambda, p)=1$. Then by (13) there is $[A] \in B(k)_{p}$ with

$$
\operatorname{inv}_{\wp}([A])= \begin{cases}\lambda / p^{i}, & \wp=\wp_{0}, \\ -\lambda / p^{i}, & \wp=\wp_{1}, \\ 0, & \text { otherwise. }\end{cases}
$$

We now claim that $[A] \in B(K \mid k)_{p} \cap B\left(K^{\prime} \mid k\right)_{p}$.

Proof. By Theorem 3 we have, for all $\wp \in P(k)$,

$$
\begin{aligned}
\min \left\{\nu_{p}\left(f_{K^{\prime} \mid k}(\mathcal{P})\right) \mid \wp \in\right. & \left.P\left(K^{\prime}\right) \wedge \mathcal{P} \mid \wp\right\} \\
& \geq \min \left\{\nu_{p}\left(f_{K \mid k}(\mathcal{P})\right)|\wp \in P(K) \wedge \mathcal{P}| \wp\right\} .
\end{aligned}
$$

By definition $[A]$ is contained in $B(K \mid k)_{p}$, because all divisors $\mathcal{P}$ of $\wp_{1}$ in $K$ satisfy $\nu_{p}\left(f_{K \mid k}(\mathcal{P})\right) \geq i$; the same holds for the divisors of $\wp_{0}$ in $K$. Hence (16) yields $[A] \in B\left(K^{\prime} \mid k\right)_{p}$.

Now we have $\overline{[A] \cdot\left[B_{\iota_{0}}\right]}=\overline{\left[B_{\iota_{0}}\right]}$ and we can construct a new element $\left[B_{\iota_{0}}^{\prime}\right]$ which represents the coset of $\left[B_{\iota_{0}}\right]$ with

$$
\operatorname{inv}_{\wp}\left(\left[B_{\iota_{0}}^{\prime}\right]\right)=0 \quad \text { for all unramified } \wp \in P(k) \backslash\left\{\wp_{0}\right\} .
$$

Since $\left[B_{\iota_{0}}\right]$ is uniquely determined by local invariants and because of the simple form of $B(\mathbb{R}), B(\mathbb{C})$ the meaning of $(17)$ is that there are only finitely many cosets.

The author wants to thank the referee for his most useful comments. 


\section{References}

[1] J. W. S. Cassels and A. Fröhlich (eds.), Algebraic Number Theory, Academic Press, 1967.

[2] B. Fein, W. Kantor and M. Schacher, Relative Brauer groups II, J. Reine Angew. Math. 328 (1981), 374-403.

[3] W. Feit, Some consequences of the classification of finite simple groups, in: Proc. Sympos. Pure Math. 37, Amer. Math. Soc., 1980, 175-181.

[4] S. Gurak, On the Hasse norm principle, J. Reine Angew. Math. 299/300 (1978), $16-27$.

[5] R. Guralnick, Zeroes of permutation characters with applications to prime splitting and Brauer groups, J. Algebra 131 (1990), 294-302.

[6] W. Jehne, Kronecker classes of algebraic number fields, J. Number Theory 9 (1977), 279-320.

[7] -, On Kronecker classes of atomic extensions, Proc. London Math. Soc. (3) 34 (1977), 32-64.

[8] N. Klingen, Zahlkörper mit gleicher Primzerlegung, J. Reine Angew. Math. 299/300 (1978), 342-384.

[9] M. Lochter, Neue zahlentheoretische Aspekte der Kronecker-Äquivalenz, thesis, Köln, 1992.

[10] - New characterizations of Kronecker equivalence, J. Number Theory, to appear.

[11] -, Weakly Kronecker equivalent number fields, Acta Arith., to appear.

[12] W. Narkiewicz, Elementary and Analytic Theory of Algebraic Numbers, 2nd ed., Springer, Berlin, 1990.

[13] J. Neukirch, Class Field Theory, Springer, 1986.

[14] R. S. Pierce, Associative Algebras, Springer, Berlin, 1982.

[15] J. P. Serre, Corps locaux, Hermann, Paris, 1968.

[16] L. Stern, On the norm groups of global fields, J. Number Theory 32 (1989), 203-219.

[17] - On the equality of norm groups of global fields, ibid. 36 (1990), 108-126.

[18] B. L. van der Waerden, Die Seltenheit der Gleichungen mit Affekt, Math. Ann. 109 (1934), 13-16.

FB 9, MATHEMATIK

UNIVERSITÄT DES SAARLANDES

66041 SAARBRÜCKEN

GERMANY

Received on 31.12.1992

and in revised form on 30.8.1993 